Article

\title{
Genome-Wide Identification of WOX Gene Family and Expression Analysis during Rejuvenational Rhizogenesis in Walnut (Juglans regia L.)
}

\author{
Yingying Chang ${ }^{1}$, Xiaobo Song ${ }^{1}$, Qixiang Zhang ${ }^{2}$, Hao Liu ${ }^{3}$, Yongchao Bai ${ }^{1}{ }^{1}$, Xiashuo Lei ${ }^{1}$ \\ and Dong Pei ${ }^{1, *}$ \\ 1 State Key Laboratory of Tree Genetics and Breeding, Key Laboratory of Tree Breeding and Cultivation of \\ State Forestry and Grassland Administration, Research Institute of Forestry, Chinese Academy of Forestry, \\ Beijing 100091, China; Yingying_Chang0123@163.com (Y.C.); xiaobo.song@caf.ac.cn (X.S.); \\ baiychao@163.com (Y.B.); leixiashuo@126.com (X.L.) \\ 2 The Nurturing Station for the State Key Laboratory of Subtropical Silviculture, School of Forestry and \\ Biotechnology, Zhejiang A\&F University, Lin'an 311300, China; qixiangzh@163.com \\ 3 Tianjin Institute of Forestry and Pomology, Tianjin 300384, China; glkl@163.com \\ * Correspondence: pei.dong@caf.ac.cn; Tel.: +86-010-6288-9624
}

Received: 18 September 2019; Accepted: 16 December 2019; Published: 20 December 2019

\begin{abstract}
Rejuvenation is an efficient approach used in the cuttings of trees and horticultural crops, to improve their rooting ability, especially in difficult-to-root trees. WOX gene family members are involved in cell-fate transformation through balancing the maintenance and proliferation of the stem cells. However, there are no reports about the WOX gene family in Walnut (Juglans regia L.) and its relationship between rejuvenation and adventitious roots formation (ARF). Here, a genome-wide identification of JrWOX genes and their physical and chemical properties, phylogeny, and expression profiles in different organs and during rejuvenation-induced ARF is reported. The phenotype and histology characteristics of mature and rejuvenated cuttings (Mc and Rc) are also observed. In this study, 12 genes were identified and clustered into three groups based on phylogenetics, special domains, and conserved motifs. The gene structures and conserved motifs were relatively conserved, while the 12 sequences of the JrWOXs domain were diversified. Gene expression in root, stem, leaf, female flower, immature fruit, and zygotic embryo revealed that the expression levels of $\operatorname{JrWOX} 4 a$, $J r W O X 4 b, J r W O X 5, J r W O X 11$, and JrWOX13 in the root were significantly higher than those of other JrWOXs, while only the expression of JrWOX11 was exclusive to the root organ. Additionally, rejuvenation treatment significantly induced almost all $\mathrm{JrWOX}$ genes, except $\mathrm{JrWOX} 4 a$, JrWOX4b, and JrWOX13 (Rc 0 vs. Mc 0). During the ARF process, the transcripts of JrWOX11 and JrWOX5 were consecutively increased on a significance level; in contrast, the transcription levels of the other JrWOXs decreased or changed insignificantly. The phenotype and histology observation indicate that rejuvenation treatment made the base of the stem expand and reduced the thickness and density of sclerenchyma between the cortex and phloem. This might provide the conditions for the formation of new meristem niches. The results provided insight into the JrWOX genes' general characteristics and their roles in rejuvenation-induced ARF.
\end{abstract}

Keywords: WOX family; Juglans regia; rejuvenation; ARF

\section{Introduction}

Adventitious root formation (ARF) is a common and widespread adaptive trait that is vital for vegetative propagation in woody plants. In some economically important trees, the poor rhizogenesis of stem cuttings has been a main bottleneck for the development of elite cultivars. To overcome the 
problems associated with the low rooting abilities of stem cuttings in difficult-to-root trees, as well as to better understand the regulation mechanism underlying rhizogenesis, extensive studies have been conducted around rejuvenation and rhizogenesis [1,2]. Rejuvenation, including serial grafting, etiolation, repeated in vitro subculturing, heavy pruning, and mound-layering [3,4], could not only regress the stem cuttings to a more juvenile state, but also significantly improve the competence of ARF. The previous research of ARF improved by rejuvenation has been carried out in terms of phenotype, microanatomy, and physiology [5-7]. However, the molecular mechanisms that regulate ARF in difficult-to-root trees are poorly understood.

WUSCHEL-related homeobox (WOX) genes, coding WOX transcription factors, are plant-specific homeobox subfamily genes and are widely found in green plants [8]. WOX genes play crucial roles in key developmental processes, by organizing stem cell maintenance and proliferation in the shoot apical meristem (SAM), root apical meristem (RAM), and cambium (CAM) [8-12]. AtWUS, a member of the modern WOX clade (MC), is involved in the feedback loop with CLV3 for SAM maintenance and also regulate the floral patterning $[13,14]$. AtWOX5, mainly expressed in the quiescent center and regulates the stem cell identity of RAM via a negative feedback signal provided by CLE40 $[15,16]$. AtWOX4, is also a MC-WOX gene, primarily regulates stem cell maintenance of CAM through the CLE41/CLE44-PXY-WOX4/WOX14 pathway [17]. AtWOX1 act redundantly with PRESSED FLOWER1 (PRS1/AtWOX3) during the formation of the lateral domains of vegetative and floral organs [18]. AtWOX2 is expressed specifically in zygotes, and is required for apical patterning [19]. AtWOX6/PRETTY FEW SEEDS2 (PFS2) is expressed abundantly in developing ovules, and influence the ovule formation [20]. AtWOX8 and AtWOX9 regulate embryonic development [19,21]. AtWOX11 and 12 are involved in the first-step cell fate transition during ARF of detached leaves [22,23]. AtWOX13 and AtWOX14 are also have effects on the regulation of primary and lateral root development in Arabidopsis [24]. The roles of some WOXs in adventitious root formation and development have also been well studied in annual plant rice (Oryza sativa L.) [25-27], and the easy-to-root tree poplars (Populus tomentosa Carr) $[9,28]$. By contrast, there is less information about their mechanism in difficult-to-root trees.

Walnuts are generally recognized as being difficult-to-root species and even more difficult to propagate from cuttings. With the completion of the walnut genome sequence and multiple analysis of the rejuvenation in walnut [5,29-31], the walnut could be adopted as an important material for difficult-to-root trees to investigate the regulation mechanism in ARF. The patent created from walnut also significantly improve the competence of ARF in numerous other difficult-to-root trees, such as cork oak (Quercus suber L.), teak (Tectona grandis L.), and California redwood (Sequoia sempervirens (Lamb.) Endl.) [32]. In recent years, the transcriptome landscape of walnut has also been reported, and that gene transcriptional regulatory network is undergoing building with the RNA-seq data and bioinformatics tools during ARF by rejuvenation in walnut $[33,34]$. While two WOX genes are expressed dynamically in the process of walnut rooting [35], whether WOX genes are induced by rejuvenation or whether other WOX genes are involved in ARF of walnut trees has not been investigated.

In this study, we systematically characterized 12 WOX genes in the common walnut. The basic local alignment search tool (BLAST) was used to identify JrWOX genes from the available genome in walnut [36], thereby detailed information about the physiological and biochemical characters, structural features, and conserve motifs of the 12 genes were revealed. There might exist differences between easy-to-root trees and difficult-to-root trees. We also constructed a phylogenetic tree of WOX genes and the evolution of the root. To understand the function of rejuvenation on JrWOXs, we studied the transcriptional levels of JrWOXs in mature and rejuvenated cuttings. Subsequently, the expression profiles of JrWOXs at critical time points in ARF of mature and rejuvenated cuttings were also detected. Our results provide useful theoretical support for the functional characterization of these JrWOXs genes that are involved in the ARF, improved by rejuvenation in common walnut, and provide insight regarding the molecular mechanisms of rejuvenation in difficult-to-root trees. 


\section{Materials and Methods}

\subsection{Identification and Bioinformatics Analysis of WUSCHEL-Related Homeobox Genes in J. regia Genomes}

The genomic sequences of common walnut J. regia were first downloaded from NCBI (http://planttfdb.cbi.pku.edu.cn) [37], and the predicted WOX genes in J. regia were found in PlantTFDB (http://ftp.ccb.jhu.edu/pub/dpuiu.Walnut/English_Walnut/v.2.0d). The genomic sequences were conducted to search homologous WOX genes, using known WOX proteins from Arabidopsis thaliana as queries, with a cutoff E-value of $<10^{-10}$. Conserved Domain Search (http://www.ncbi.nlm.nih. gov/Structure/cdd/wrpsb.cgi) was performed to confirm the integrity of homeobox domain (PF00046) in these candidate proteins, and the proteins lacking HD domain were removed. Subsequently, Gene Structure Display Server (http://gsds.cbi.pku.edu.cn/), ProtParam (http://web.expasy.org/protparam/), and Plant-mPLoc (http://www.csbio.sjtu.edu.cn/bioinf/plant-multi/) were used to analyze JrWOX genes' structure, basic physical and chemical properties, and subcellular location, respectively. Alignment of canonical conserved residues in JrWOX homeodomain sequences were analyzed by WebLogo (web-based sequence logo generating application; Weblogo.berkeley.edu). Finally, MEME program was applied for the elucidation of Motifs in the candidate proteins. Each JrWOX gene was given a unique name based on the BAST results with AtWOX1-15 (Table 1).

Table 1. Basic characteristics of the proteins encoded by JrWOXs.

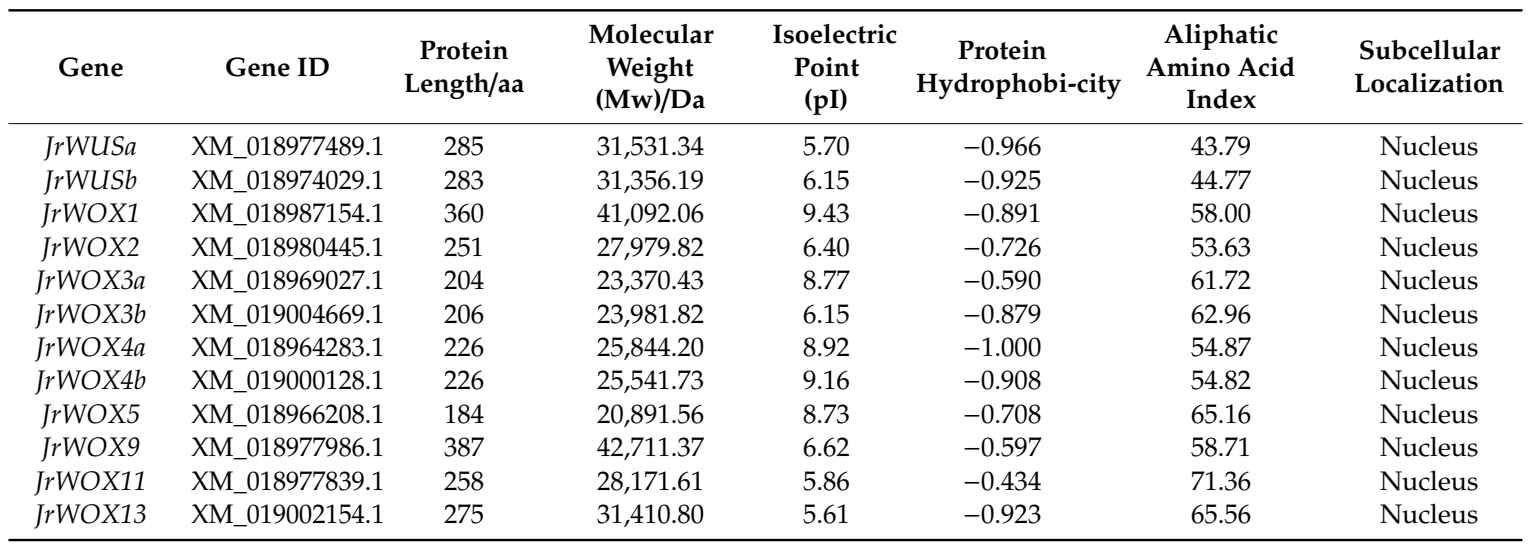

\subsection{Phylogenetic Analysis}

The JrWOX protein sequences from green alga (Ostreococcus tauri, Ot), moss (Physcomitrella patens, $\mathrm{Ph}$ ), lycophyte (Selaginella moellendorffii, Sm), fern (Ceratopteris richardii, Cr; and Cyathea australis, Ca), gymnosperm (Ginkgo biloba, Gb; Picea abies, Pa), Monocotyledon angiosperm (Oryza sativa, Os; and Zea mays, Zm), Dicotyledon angiosperm (Arabidopsis thaliana, At; Populus trichocarpa, Ptr; Spirodela polyrhiza, Sp; Quercus suber, Qs; Juglans regia, Jr), and representative species were used for phylogenetic analysis. The gene ID were listed in Table A1. Clustal X 2.0 software [38] was used for the multiple alignment, and unrooted phylogenetic trees were constructed using MEGA 7.0 software [39] with the neighbor-joining (NJ) method by 1000 bootstrap replicates.

\subsection{Analysis of Regulatory Elements in the Promoter Regions of the JrWOX Gene Family}

The upstream regions $(2.0 \mathrm{~kb}$ ) of the translation initiation sites (ATG) of WOX genes were used as promoter fragments. In addition, the elements were located in the promoter sequences, using the program PlantCARE online (http://bioinformatics.psb.ugent.be/webtools/plantcare/html/).

\subsection{Plant Materials, Growth Conditions, and Treatments}

The walnut stock cultivar "Zhongningsheng" [ZNS, (Juglans hindsii $\times$ Juglans regia)] [30], which is selected with a steady and high yield, a good adaptation, and high graft compatibility, is grown in the 
Luoning county, Henan Province. To analyze expression profiles of JrWOX genes in different organs, three 23-year-old ZNF trees were chosen for the collection of root (R), stem (S), leaf (L), female flower (FF), immature fruit (IF), and zygotic embryo (ZE) samples. The cuttings of 1-year-old ZNS shoots were rejuvenatedand rooting. Then the $\operatorname{root}(\mathrm{R})$ was collected, washed and dried. Stem $(S)$ and leaf $(\mathrm{L})$ were also obtained from the middle part of 1-year-old saplings. Female flower (FF), immature fruit (IF), and zygotic embryo (ZE) were harvested, respectively, 15, 45, and 60 days after flower, when the trees were at the stage of pollination, after pollination, and starting to swell, respectively. Six samples of each organ from the same tree were mixed as one biological replicate. All the above materials were rapidly frozen in liquid nitrogen and stored at $-80^{\circ} \mathrm{C}$.

Rejuvenated soft cuttings (Rc) of ZNS were obtained as described as follows: (1) serial grafting, the buds of new ZNS shoots were used as scions and were grafted to 1-year-old seedlings (rootstock) for sprouting; (2) etiolation, 1-year-old grafted seedlings were placed horizontally on the greenhouse seedbeds and covered with $4-5 \mathrm{~cm}$ of clean wet sand. The sand temperature and humidity were respectively held at $15 \pm 5{ }^{\circ} \mathrm{C}$ and $50 \%$, which ensured maximum side bud germination rate. After about two months, the new shoots of $14-18 \mathrm{~cm}$ height were harvested as rejuvenated soft cuttings [5]. Mature soft cuttings (Mc) of the same height were directly collected from new shoots of the 23-year-old ZNF trees. The 8-10 $\mathrm{cm}$ base of both cutting materials was treated with $9 \mathrm{mM}$ of IBA (dissolve in $30 \%(v / v)$ ethanol) for about $30 \mathrm{~s}$ and then submerged in seedbeds. Under the conditions of field, $80 \%$ relative humidity and $23 \pm 5^{\circ} \mathrm{C}$ temperature were controlled by automatic intermittent spraying. After rooting induction $0,1,2,3$, and 5 days, cambium and phloem tissues surrounding the xylem of the $1 \mathrm{~cm}$ base of the both cuttings were scraped by a sterile scalpel blade. The harvested materials were, in turn, named Rc0, 1, 2, 3, 5 days and Mc0, 1, 2, 3, 5 days, then rapidly frozen in liquid nitrogen and stored at $-80{ }^{\circ} \mathrm{C}$ to detect the role of $\mathrm{JrWOX}$ genes in rooting. Three biological replicates were performed in different pots for each treatment.

\subsection{Expression Pattern Analysis of the JrWOX Genes}

RNA isolation, quantitative reverse transcription qRT-CR, and RT-PCR were performed according to Ma et al. [33]. GAPHD was used as an endogenous control gene. Then, qRT-PCR was performed by using the SYBR Premix Ex Taq ${ }^{\mathrm{TM}}$ II Kit (TaKaRa, Dalian, China) on a LightCycler 480 system (Roche Applied Science, Penzberg, Germany) according to the manufacturer's instructions. The primers used for qRT-PCR were designed by using Primer 5.0 software (PREMIER Biosoft International, Palo Alto, CA, USA) and listed in Table A2. All experiments were carried out at least three times, and data analysis was performed by using the $2^{-\Delta \Delta \mathrm{Ct}}$ method [40].

\section{Results}

\subsection{Identification and Sequence Characteristics of JrWOX Genes}

To identify WOX genes in J. regia, the protein sequences of 15 A. thaliana WOX (AtWOX) were used as a query to perform basic local alignment search tool algorithms (BLASTP) and Hidden Markov models (HMMs) search against the J. regia genome database. A total of 12 nonredundant proteins were identified, and protein length, molecular weight $(\mathrm{Mw})$, isoelectric point $(\mathrm{pI})$, protein hydrophobicity, aliphatic amino acid index, and subcellular localization prediction are shown in Table 1. The length of proteins varied from 184 aa (JrWOX5) to 387 aa (JrWOX9), the predicted pI ranged from 5.61 to 9.43 , and the Mw ranged from 23.37 to $42.71 \mathrm{kDa}$ (Table 1). The protein hydrophobicity is $<0$, indicating that the 12 JrWOX proteins are hydrophilic. Aliphatic amino acid index is $>40$, indicating that JrWOX proteins are thermally stable. All the 12 WOX proteins are predicted to localize in the nucleus based on the signal peptides. JrWOXs act as a transcription factor in the nucleus, regulating the expression of the downstream target genes. 


\subsection{Gene Structure, Conservative Domain, and Motif Analysis of JrWOXs}

To gain further insight into the structural diversity of different groups of JrWOX genes, the simplified neighbor-joining (NJ) phylogenetic tree of their homeobox domains was constructed, as well as the analysis of gene structures and functional motifs (Figure 1). As shown in Figure 1A, the 12 JrWOX members are divided into three subfamilies. The members close on the phylogenetic tree shared similar gene structures and intron phases (Figure 1B). Extron number ranged from 2 to 4 . All members have the homeobox domain, while only the members clustered in the first subfamily (WC subfamily) have the transcription activation domain WUS-box. Three members of the nine WC-WOX also contained an ethylene-response-factor-like domain (ERF-like)_JrWUSa, JrWUSb, and JrWOX5. The same subfamily also shared a similar motif-structure, as shown in gene structure (Figure 1C). There were ten conserved motifs identified in JrWOXs; among them were motif1 and 2, and motif3 and 7 might correspond to conservative functional elements distributing in homeobox, WUS-box and ERF-like domains, respectively. JrWOX13 only contained the common conserved motif1 and 2; however, IC-subfamily members JrWOX9 and JrWOX11 contained an additional Motif5 at the C-terminus. In regard to WC-WOXs, JrWUSa/b specifically contained motif4, while JrWOX4a/b exclusively contained motif5, 9, and 10. These results suggested that gene structures and formation of motifs are associated with the sub-functionalization and neofunctionalization of WOX members.

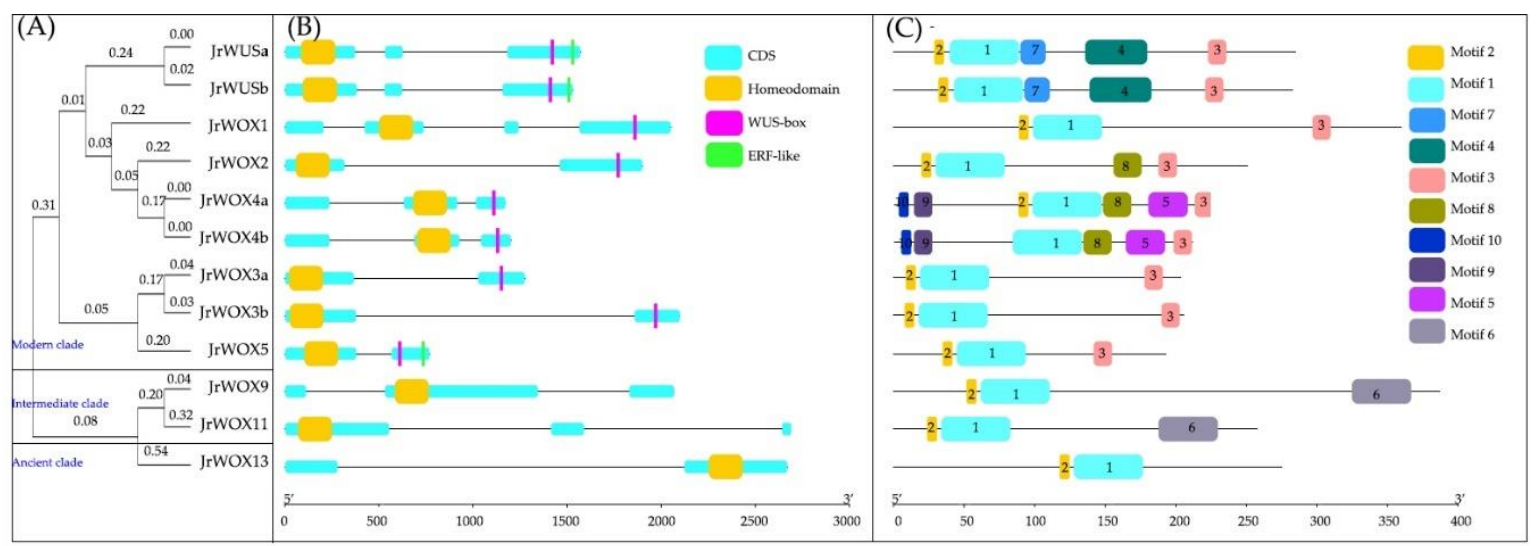

Figure 1. Phylogenetic relationships, gene structure, and motif phases of the WOX genes in J. regia. (A) Phylogenetic tree based on JrWOXs homeobox domains. (B) The gene structures and intron phases of JrWOXs gene. (C) The distribution profiles of conserved motifs on JrWOXs proteins.

\subsection{Multiple Alignment the WOX Protein Sequence in J. regia}

Members of the WOX family contain a conserved homeodomain, which consists of the "helix-loop-helix-loop-helix" (Figure 2A). Alignment of the homeodomains of 12 WOX genes in $J$. regia revealed the residues were highly conserved. There were 16 highest conserved residues in the homeo-domain; among them, Leucine (L), Valine (V), and Tryptophan (W), which are marked with asterisks, are also the most conserved of the other 50 species. These three residues are located inside the structure of the homeodomain, and they might influence the factor function by changing the domain structure. The residues within the two black boxed motifs have positional correlation, which may also influence the protein function [9]. The red boxed residues within helix 3 can distinguish the three clades of WOX proteins (Figure 2A): The ' $\mathrm{YN}$ ' to 'FY' change in the sequence occurred during the evolution from the ancient to the intermediate clade, and the ' $\mathrm{R}$ ' to ' $\mathrm{H}$ ' change may have occurred during the evolution from the intermediate clade to the MC. Only the members of MC harbor the WUS-box and the EAR-like domain appeared exclusively in JrWUSa/b and WOX5 (Figure 2B,C). Interestingly, C-terminal region of IC-WOXs contained a specific motif, while there were none found in MC and AC. There are other conservative structural domains in the JrWOX family, while the functions need to be further studied. 
(A) Homeodomain

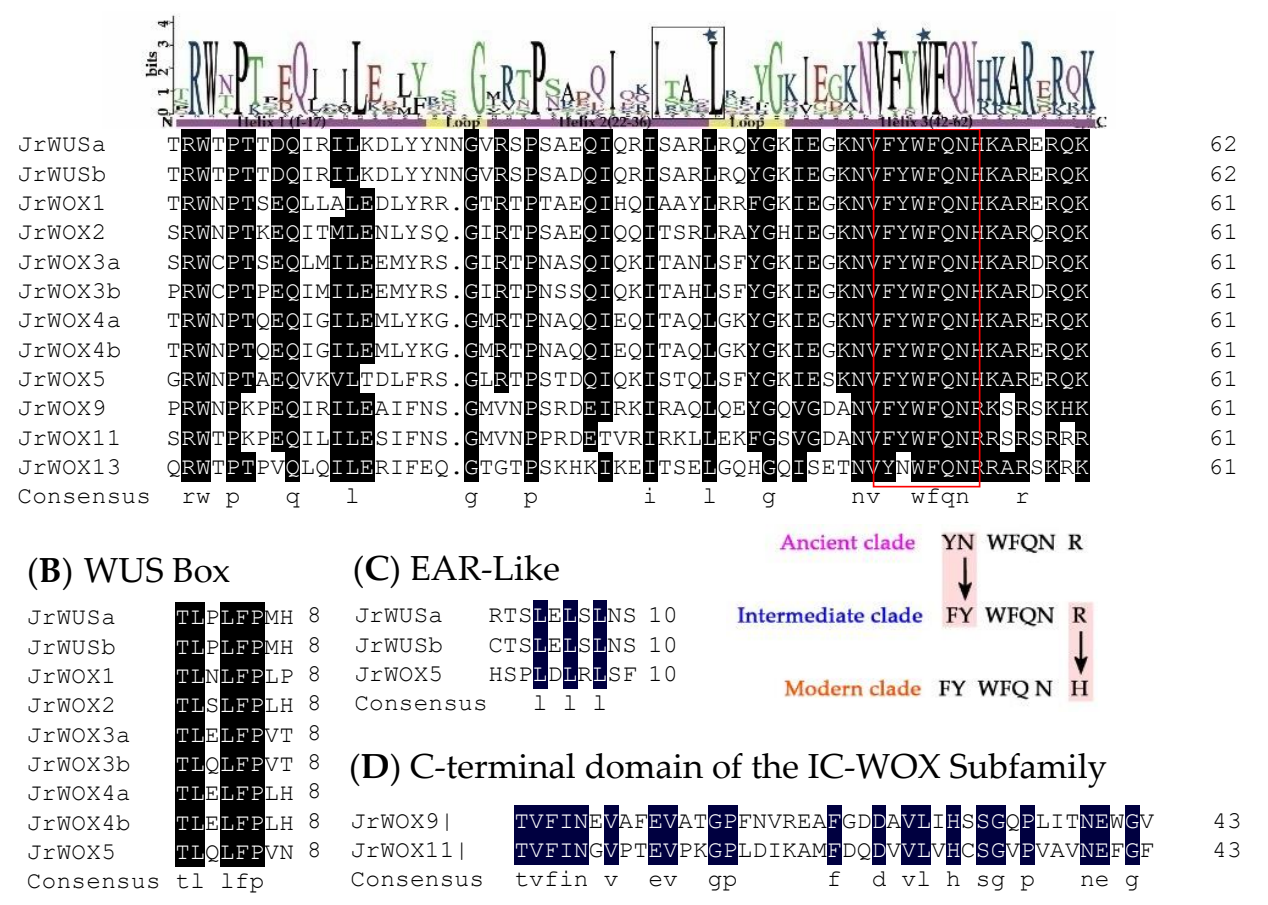

Figure 2. Comparison of the WOX protein family in J. regia. (A) Alignment of the homeodomain sequences. Asterisks indicate residues that are highly conserved in homeodomains (Gehring et al., 1990). (B) Alignment of the WUS-box that is located downstream of the homeodomain. (C) Alignment of the EAR-like domain of the MC-WOXs. The identical residues are in black boxes. (D) Alignment of the C-terminal region in a subset of IC-WOX subfamily.

\subsection{Characterization of Cis-Acting Elements in the Promoter Regions of JrWOXs}

To further investigate the possible regulation mechanism of WOX genes in J. regia, the cis-elements in the promoter regions of JrWOX genes were analyzed, using the PlantCARE database. A series of hormone-related (e.g., Auxin, ABA, ZT, GA, MeJA, and Ethylene), and development-related (e.g., meristem expression, seed-specific regulation, endosperm expression, circadian control, and palisade mesophyll cells), stress-related (e.g., heat, drought, low temperature, and anaerobic induction), and light response cis-acting elements were identified (Figures 3 and A1). In hormone-related cis-acting elements, $58.33 \%$ of JrWOXs (seven genes) had the auxin response elements, $91.67 \%$ of JrWOXs (11 genes) had the ABA response elements, which was the most abundant cis-acting element, with its number reaching 54. Moreover, as-1 (Plant hormone responsiveness), O2-site (zein metabolism regulation), ERE (ethylene responsiveness), CGTCA-motif (MeJA-responsiveness), GARE-motif (MeJ-responsiveness), TCA-element (salicylic acid responsiveness), P-box (gibberellin responsiveness), TATC-box (gibberellin responsiveness), GARE-motif (gibberellin responsiveness), TGA-element (auxin responsiveness), and AuxRR-core (auxin responsiveness) were identified in the promoters of 8, 8, 5, 8, 8, 10, 5, 3, 3, 6, and 1 JrWOXs, respectively. In development-related cis-acting elements, there were 10 root-specific elements (W-box), seven seed-specific regulation elements (RY-element), eight endosperm-specific expression elements (GCN4_motif and AACA_motif), eight meristem-specific regulatory (CAT-box and CCGTCC motif), and two circadian cis-acting elements, that were located in 7, 4, 6, 6, and $2 \mathrm{JrWOXs}$ promoters, respectively. Furthermore, large numbers of cis-acting elements related to drought resistant (MRE, MBS, and DRE), abiotic resistant (ARE, STRE, WRE, and GC-motif), low temperature resistant (LTR), etc., were detected. This result implied that JrWOXs might not only be involved in the developmental processes, but might also be recruited in biotic and abiotic stress. 


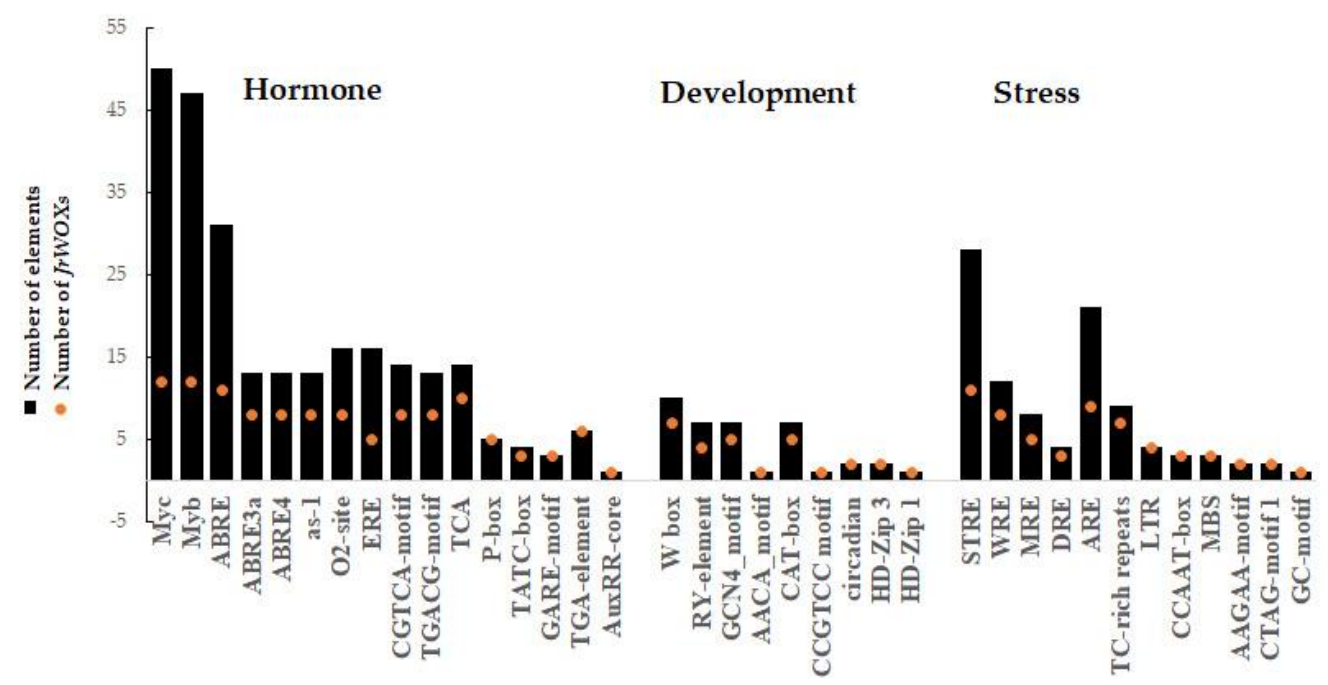

Figure 3. Various cis-acting elements in the promoter of JrWOX genes.

\subsection{Phylogenetic Analysis of J. regia WOXs and Root Evolution in Green Plants}

To further investigate the possible function of JrWOXs during evolutionary change, a phylogenetic tree of 138 WOX proteins from 14 species was conducted, using the neighbor-joining (NJ) method. The phylogenetic results show that all the WOX proteins were allocated into three clades: the modern clade (MC), the intermediate clade (IC), and the ancient clade (AC) (Figure 4A). Green algae and mosses, having no root structures, are only distributed with AC-WOX genes. The WOX gene may originate from green algae, as WOX genes can be found in the genomes of euphyllophytes, lycophytes, moss, and green algae, but do not exist in the red algae genome [13,41]. S. kraussiana encodes four WOX proteins in AC and one WOX protein in IC; it is worth noting was that there were two lycophyte-specific SkWOX proteins, SkWOX11A and SkWOX11B, marked with a purple dotted line in Figure 4A. SkWOX11A and SkWOX11B appear to be transitional between the AC and IC, in line with the fact that the first root-evolution event was in the lycophytes [42]. The second root-evolution event was in the ancestor of the ferns. Although CrWUL were included in the WC, they might represent a transitional evolutionary stage from IC [43]. This suggests that IC WOX proteins are recruited in adventitious and lateral root formation. The WC genes have further evolved in gymnosperms and angiosperms. During the seed plant stage, primary roots appeared in addition to adventitious and lateral roots (Figure 4B). In J. regia, 12 WOXs were also classified into three clades: as for AC, JrWOX13 are homologous to Arabidopsis WOX10, WOX13, and WOX14. JrWOX9 and JrWOX11 are homologous to Arabidopsis WOX8, WOX9, WOX11, and WOX12 belonging to IC. The WUS Clade contained nine JrWOX genes, including JrWUSa, JrWUSb, JrWOX1, JrWOX2, JrWOX3a, JrWOX3b, JrWOX4a, JrWOX4b, and JrWOX5. This indicated that the differentiation of transcription factor family members may be earlier than that of seed species. However, the possible function of WOX family members during root morphological variation in seed plants, especially woody plants, is not enough. 


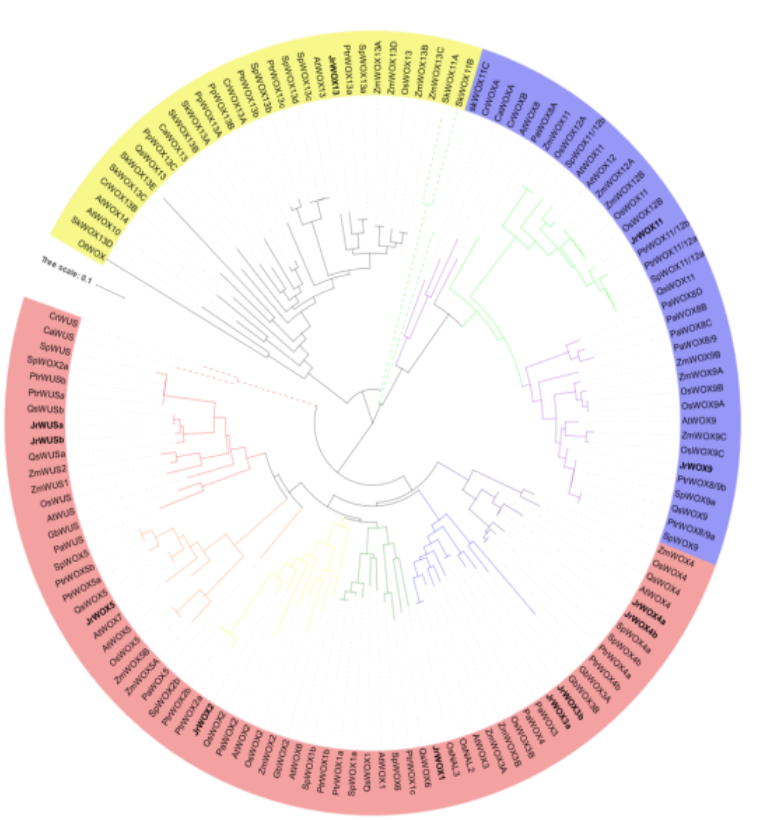

(A)

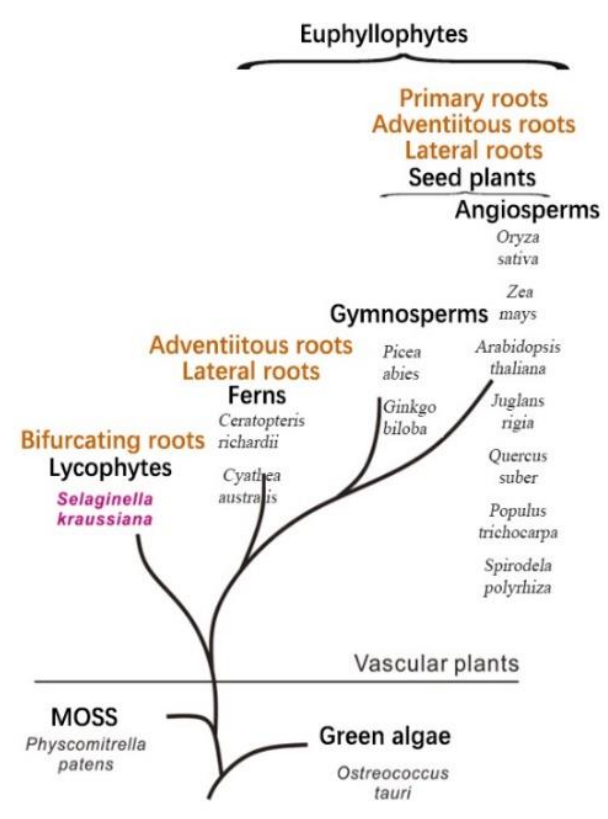

(B)

Figure 4. Dendrogram based on the full-length protein sequences of the WOXs and root evolution in green plants. (A) Phylogenetic analysis of WOXs. Bootstrap values are given (1000 rounds). The tree contains sequences from green alga (Ostreococcus tauri, Ot), moss (Physcomitrella patens, $\mathrm{Ph}$ ), lycophyte (Selaginella kraussiana, Sk), fern (Ceratopteris richardii, $\mathrm{Cr}$; and Cyathea australis, Ca), gymnosperm (Ginkgo biloba, Gb; Picea abies, Pa), and Monocotyledon angiosperm (Oryza sativa, Os; and Zea mays, Zm) and Dicotyledon angiosperm (Arabidopsis thaliana, At; Populus trichocarpa, Ptr; Spirodela polyrhiza, Sp; Quercus suber, Qs; Juglans regia, Jr) representative species. The unrooted amino acid sequence similarity trees were generated by using the MEGA7.0 software by the neighbor-joining method. Modern clade (red; M); intermediate clade (blue; I); ancient clade (black; A). (B) Simplified evolutionary route of green plants, showing two existing branches of vascular plants; lycophytes and euphyllophytes.

\subsection{Rejuvenation-Changed Phenotype and Histological Features of Cuttings}

The leafy twigs were used as cuttings for detecting morphological characteristic changes. The bark of mature cuttings (Mc) is a uniform green (Figure 5A). The base of the rejuvenated cuttings (Rc) became swollen, and the bark was yellowish-white, while the upper bark was also yellowish-white (Figure 5B).

Histological observation of mature and rejuvenated cuttings showed that a large number of histologic sections indicated that there was no latent rooting primordial group in Mc and Rc, implying walnut rooting in a type of induction model. The sections of Mc and Rc both consisted of periderm, cortex, cortical vascular tissue, and pith. Interestingly, the sclerenchyma distributing between the cortex and phloem was arranged more closely in Mc and contained more layers of cells than that in Rc (Figure 5C,D), which might be one of the histological reasons for rejuvenation improving the rooting ability of walnut cuttings. 

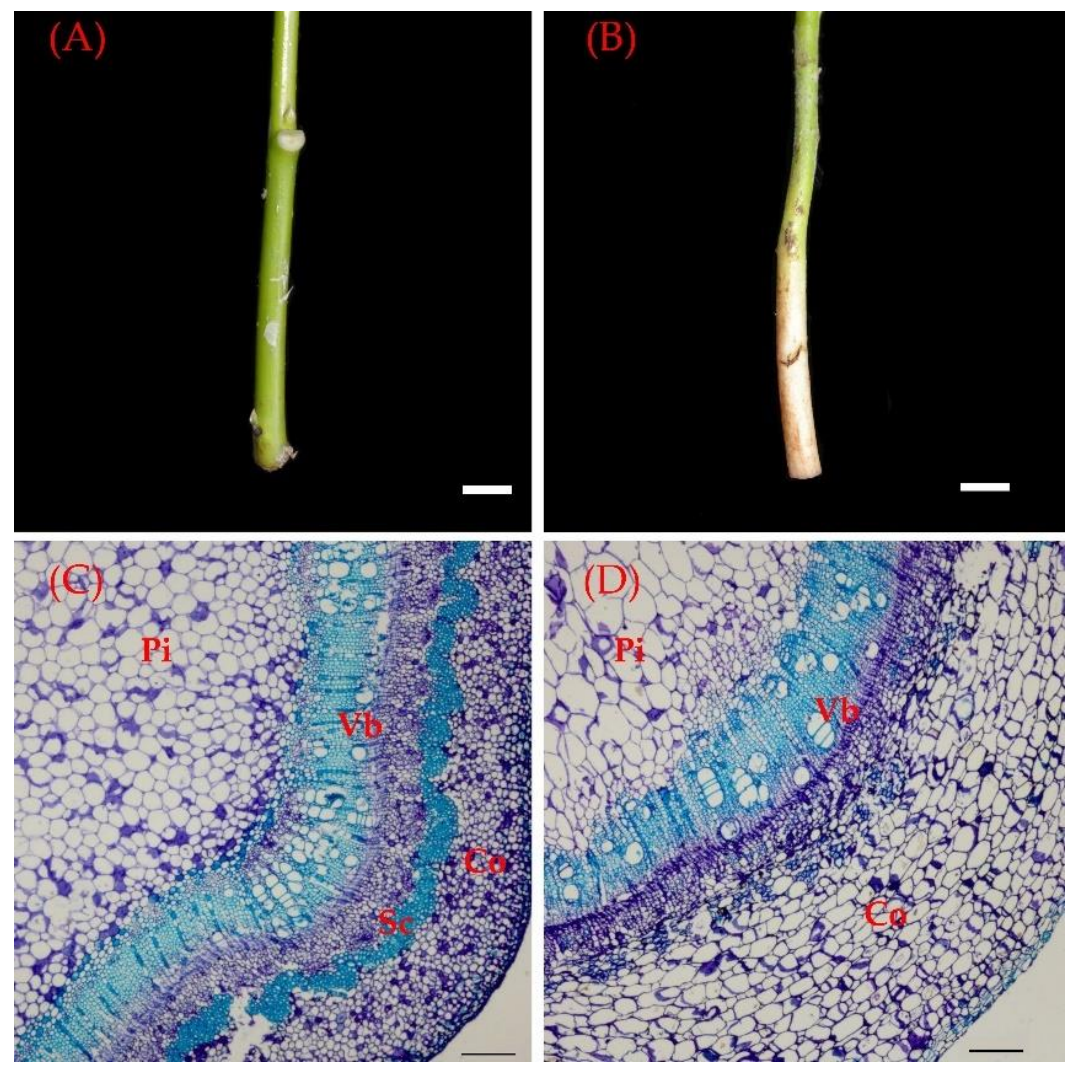

Figure 5. Phenotype and histological comparison between mature cuttings and rejuvenated cuttings. $(\mathbf{A}, \mathbf{C})$ Phenotype and histological investigation of Mc. (B,D) Phenotype and histological investigation of Rc. Co, cortex. Vb, cortical vascular tissue. Pi: pith. Sc: sclerenchyma. Scale bars: $10 \mathrm{~mm}(\mathbf{A}, \mathbf{B})$, $100 \mu \mathrm{m}(\mathbf{C}, \mathbf{D})$.

\subsection{Diversified Expression Patterns of JrWOX Genes}

\subsubsection{Expression Patterns of JrWOX Genes in Diversified Organs}

To gain insights into possible developmental and physiological functions of JrWOX proteins, the expression pattern of WOX genes in ZNS were analyzed by semi-quantitative RT-PCR and relative quantitative RT-PCR (Figure 6). The results showed that the performances of 12 family members were different in different organs. In WC, JrWUSa/b, JrWOX1 and JrWOX2 were expressed specifically in the zygotic embryo (ZE). There were slightly different expression profiles between two subgenes. The highest expressions of $J r W O X 3 a$ and $J r W O X 3 b$ were in female flowers (FF) and zygotic embryos (ZEs), respectively, although they both had lower expressions in the root (R), stem (S), leaf (L), and immature fruit (IF). The expression level of $J r W O X 4 a$ and $J r W O X 4 b$ were both higher in vegetative organs than reproductive organs; however, the highest expressions of JrWOX4a and JrWOX4b were in the root (R) and stem (S), respectively. Similar to PtoWOX5b, JrWOX5 was ubiquitously expressed, but AtWOX5 was restricted to the RAM $[15,44]$. In IC, JrWOX9 was expressed highest in female flowers (FF), while the highest expression of JrWOX11 was in the root (R) system. The only subclass of the ancient WOX clade in J. regia, JrWOX13, also exhibited a ubiquitous expression pattern. 

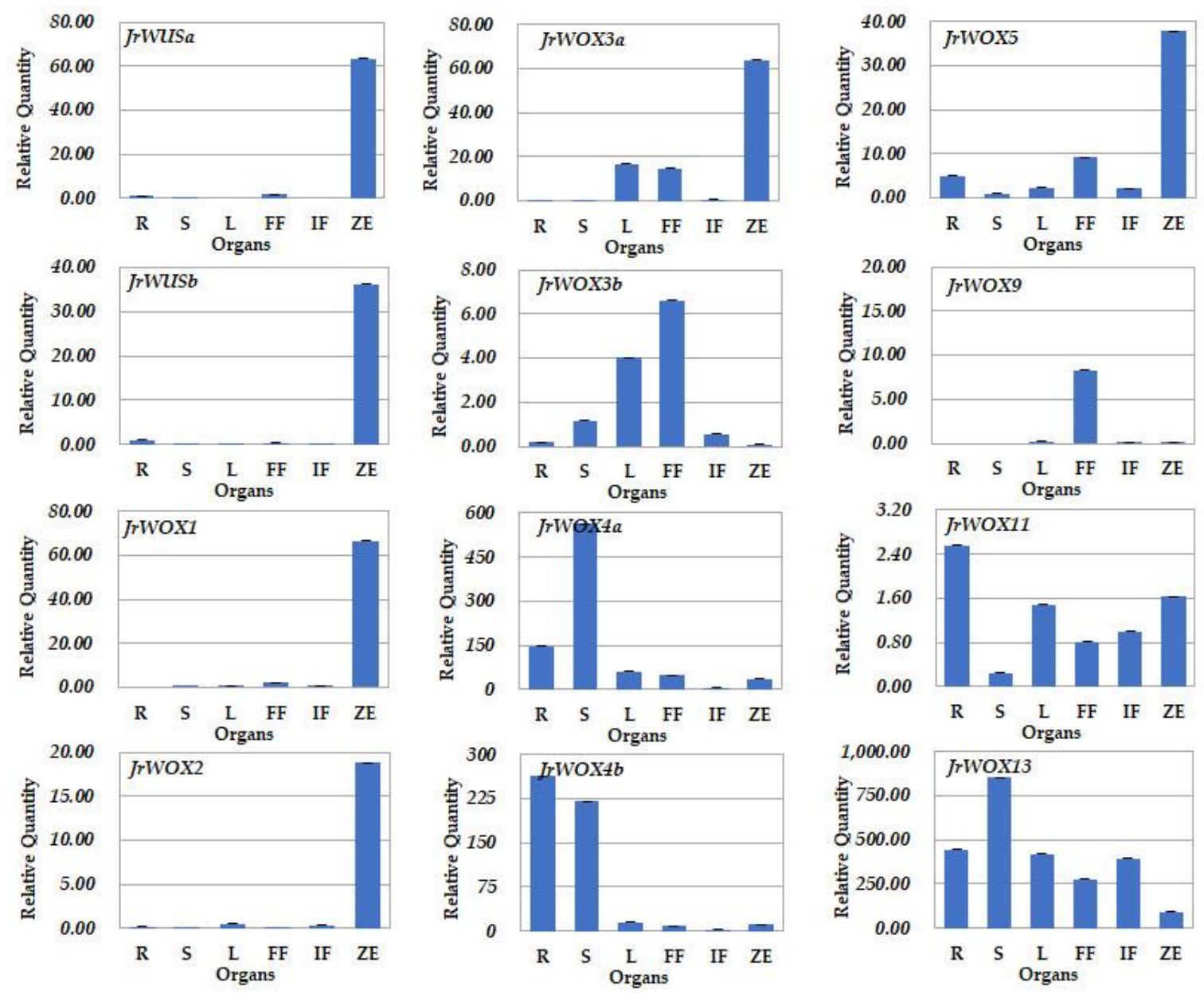

Figure 6. Expression analysis of walnut WOX family's genes in different tissues, using qRT-PCR.

\subsubsection{Expression Patterns of JrWOX Genes in ARF of Mature and Rejuvenated Cuttings}

Rejuvenation is an essential factor to improve the soft-shoot rooting ability of mature woody species. In this study, the rejuvenated and mature cuttings of walnut were used as materials. The results of quantitative analysis indicated that rejuvenation increased the expression level of $J r W O X$ genes, other than JrWOX4a, JrWOX4b, and JrWOX13. In the mature cuttings, JrWUSa, JrWUSb, JrWOX1, JrWOX2, JrWOX3a, JrWOX3b, and JrWOX5 nearly did not express during the process of ARF. In the rejuvenated cuttings, JrWUSa/b, JrWOX1, JrWOX2, JrWOX3a/b, and JrWOX4a/b showed a slender declining trend, while JrWOX5 exhibited a significant increase trend. The expression level of JrWOX11 sharply increased before 2 days, and then it descended in the rejuvenated cuttings; however, in mature cuttings, JrWOX11 displayed high expression until the last rooting stage (Figure 7). The results of histology show that the cambium cells started to divide strongly three days after rejuvenated cuttings and induced rooting [5]. Then, cambium thickened, and the root meristem formed on the fourth day [5]. It can be presumed that JrWOX11 was essential for adventitious root primordia formation, and JrWOX5 was crucial for root development. 

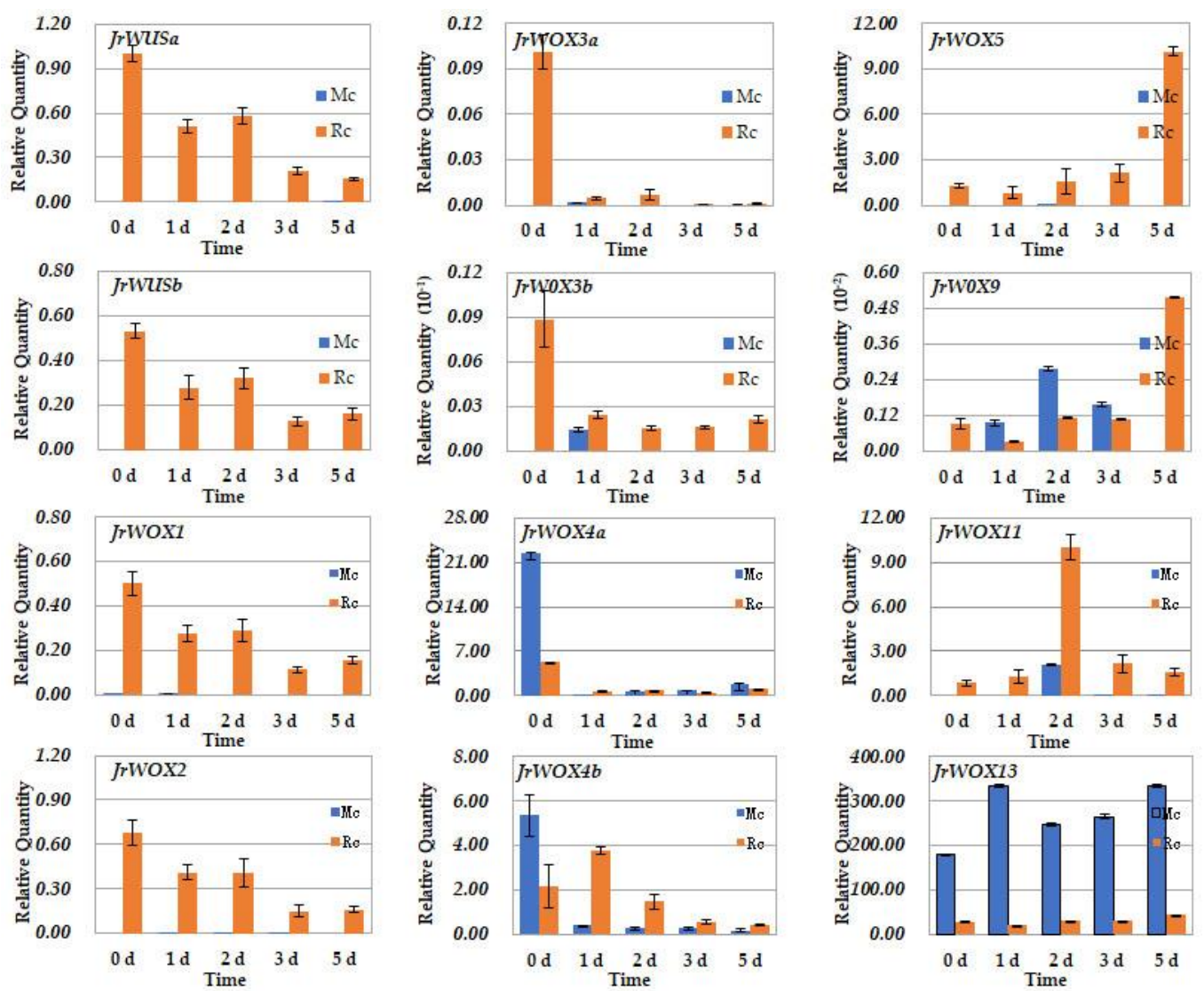

Figure 7. Expression analysis of walnut WOX family genes during adventitious roots formation in rejuvenated and mature soft shoots.

\section{Discussion}

\subsection{Diversification of WOX Genes in J. regia}

With the rapid development of sequencing technology and the increase in the efficiency and accuracy of sequencing, more and more species' genome and transcriptome data have been reported. This provides good data resources for analyzing the gene structure and function prediction on the scope of whole-genome identification and comparison. The genomes of plants are different; for example, O. tauri, P. patens, S. kraussiana, P. trichocarpa, Z. mays, A. thaliana, and O. sativa genome are 14,472 , 115, 423, 2300, 125, and $389 \mathrm{Mb}$, respectively. These plants contain WOX genes 1, 3, 8, 18, 18, 15, and 14, respectively. It could be speculated that the genome size was not the main determinant of the number of gene families [45]. Along with this, the common walnut genome is $667 \mathrm{Mb}$, while 12 WOX encoding genes were identified in J. regia (Table 1), which could be categorized into modern, intermediate, and ancient clades. While there were no JrWOX genes classified together with AtWOX6, AtWOX7, AtWOX10, or AtWOX14, other WOX genes gathered in the same clade may perform similar functions. For example, WUS-box in WOX6 and WOX7 is involved in leaf-blade outgrowth and floral organ development in Nicotiana Sylvestris [46]. In A. thaliana, while the role of AtWOX14 is unknown, it may be restricted to the early stages of lateral root formation and the floral transition, as the AtWOX13 gene is involved in replum formation during fruit development $[24,47]$. This study also identified two copies of JrWOXs which could be classified as WUS (JrWUSa and $b$ ), WOX3 (JrWOX3a and $b$ ), and WOX4 (JrWOX4a and $b$ ), and this might result from chromosomal duplication events. 


\subsection{The Characteristics of the WOX Gene Family of Walnut}

Similar physical and chemical properties indicate that members may have some similar functions. In A. thaliana and P. trichocarpa, WOX proteins' length range from 122 aa (AtWOX7) to 378 aa (AtWOX9) and from 181 aa (PtrWOX5) to 390 aa (PtrWOX8/9a). Similarly, the length of JrWOX proteins varied from 184 aa (JrWOX5) to 387 aa (JrWOX9). Because JrWOX5 and PtrWOX5 are homologous to AtWOX7, JrWOX5 may perform the same functions as PtrWOX5 and AtWOX7 in adventitious root formation and development $[44,48,49]$. Although the physicochemical properties of different JrWOX proteins were different, the sister copies of the same JrWOX protein were similar (Table 1). Proteins normally perform their biological functions depending on their own functional domains. Through protein structure analysis, it was found that all the 12 WOX transcription factor family members in walnut contained a conserved homologous domain composed of 60 amino acid residues (Figures 1B and 2A). Comparing these with the 350 WOX proteins from 50 species, homeodomain has the structure of "helix-loop-helix-turn-helix", which plays an important role in DNA recognition and binding [10]. Homeodomain contains many highly conserved amino acid residues, among which the last amino acid residue L (leucine) of the second helix structure, the last amino acid residue I/V (isoleucine/valine) of the turn structure, and the amino acid residue $\mathrm{V}$ (valine) of the middle position of the second helix structure are the most conserved (Figure 2A). Three-dimensional structure imaging shows that these three amino acid residues are located in the interior of homeodomain, indicating that they play a key role in the function of the WOX transcription factor [46]. Moreover, the angle of formation of "helix-turn-helix" structure in ancient branches is smaller than that of intermediate clades and modern clades, which may lead to functional changes in the evolution process of WOX transcription factor. In this study, the three residues were also highly conserved in the homeodomain of the JrWOX protein family (Figure 2A). The residues marked by two black boxes (Figure 2A) may highly correlate with each other and play a role in the evolutionary process. In the homeodomain structure, there were other residues marked by two red boxes (Figure 2A); the "YN" to "FY" change in the sequence occurred during the evolution from the ancient to the intermediate clade, and the " $R$ " to " $\mathrm{H}$ " change may have occurred during the evolution from the intermediate clade to the modern clade $[41,50]$. In addition, the WUX-box domain containing "LXL" might have a transcriptional inhibitory activity and plays an important role in leaf development and flower formation $[46,50]$ (Figure 2B). The EAR-like domain may supplement the transcriptional inhibition function of WUS-box (Figure 2C). There was a specific motif in the C-terminal region of IC-WOXs (Figures 1C and A1), and multiple sequence alignment showed that this fragment was conserved (Figure 2D). Other studies, accordingly, have found that IC-WOX genes play a role in root evolution $[12,42,51]$.

\subsection{The Promoter Regions of JrWOXs}

As the binding sites of transcription factors, cis-acting elements in the promoter of the gene determine its expression patterns. In our study, a series of hormone-, development-, and stress- related cis-acting elements were detected in the promoter of JrWOXs (Figure 3). The maximum number of cis-acting elements were Myb elements, which play a role in controlling plants' secondary metabolism and regulating cell morphological formation and signal transduction pathways [52]. Previous reports have testified that auxin directly induces AtWOX11 expression, owing to the AuxRE (TGTCTC) in the promoter of AtWOX11 [13]. OsWOX11 interacting with ERF3 can promote crown root through the cytokinin signaling pathway [25]. In poplar, PtoWOX11 improves drought resistance by interacting with AP2 [53]. In difficult-to-root trees, exogenous hormones, such as NAA and IBA, are often used for rooting induction [54]. Sometimes, however, even if exogenous auxin is applied to the cuttings, they cannot take root, such as walnut, Quercus species, and teak. It can be speculated that only the supplement exogenous auxin is not enough for difficult-to-root trees. The relationship of supplement exogenous auxin, rejuvenation and protein-protein interactions in rooting is a question deserving of further research. There were also a number of organ-specific cis-elements in JrWOXs promoters, such as ACGT-core, CANNTG-motifs, GATA-motifs, and W-box ((T)TGAC(C)). Among them, ACGT, 
CANNTG, and GATA act as transcription-factor binding sites for other transcription factors that are specifically expressed in roots [55]. This suggests that JrWOXs might be involved in biotic and abiotic stress responses and plant development under regulation of these cis-acting elements.

\subsection{The Evolution of JrWOX Genes and Root-Evolution Events}

Based on phylogenetic analysis, green alga and moss only harbor ancient clade WOX genes, subsequently, intermediate clade WOX genes present in the vascular species lycophyte (Figure 4A.). In gymnosperms, the phylogenetic classification and the developmental role of WOX genes have not been clearly characterized [56]. As WOX genes from angiosperm species, such as O. sativa, Z. mays, A. thaliana, P. trichocarpa, S. polyrhiza, and Q. suber, were separated into three distinct clades-ancient clade, intermediate clade, and modern clade-12 JrWOXs were also separated into the three clades (Figure 4A.). Both Quercus and Juglans are difficult-to-root trees for low adventitious root ratio from stem cuttings [5,57]. The evolutionary distance of JrWOXs and QsWOXs were near to each other, implying that they are closely related. Motif analysis shows that a Motif 7 and Motif 3 tandem repeats in QsWOX11 and JrWOX4a/b, respectively (Figure A1). This provides clues for mechanism research on the AR formation of difficult-to-root trees.

For the evolution of the root, there is no differentiation of root in green alga and moss (Figure 4B). Fossil evidence and the root anatomy indicate that at least three root-evolution events happened in lycophytes, ferns, and seed plants, sequentially (Figure 4B) [42]. Accordingly, the morphology of roots evolved from bifurcating roots to adventitious roots and lateral roots, and then to primary roots. The evolution of roots in vascular plants is beneficial to the successful adaptation to different environments. There are more different root types in seed species, e.g., tap root system, fibrous root system, mycorrhiza, etc. The investigation of WOX genes and their functions in the polymorphic root organogenesis will help to improve the understanding of the molecular bases of root evolution. WOX genes not only influence root formation; for example, AtWOX11 and 12 are involved in the first-step cell-fate transition during de novo root organogenesis, but also influence the AR number, relative strength, and histomorphology [21,58]. In walnut, endogenous hormone distribution could be changed by rejuvenation [5]; however, whether WOX genes are involved in these changes is unclear. Furthermore, morphological observation of the difficult-to-root trees and easy-to-root trees may provide guidance for improving agricultural production. It has been considered that the distribution of thick-walled tissues between phloem and cortex determines the degree of rooting difficulty to some extent, and that thick-walled tissues do not exist or are discontinuously distributed in the easy-to-root trees, while they are arranged in a circular pattern in the difficult-to-root trees with large thickness [59]. Current studies suggest that sclerenchyma is not a physical barrier to adventitious root formation, but rather a barrier to the initiation of adventitious root primordia [60]. In walnut, breaking through the barrier around the cross-sectional areas of the cambium and phloem is an important reason for the improvement of rooting rate.

\subsection{Expression Patterns of JrWOX Genes}

The tissue expression patterns of genes in different plants can be used to identify the functions of genes. In A. thaliana and P. tomentosa, the expression patterns of WOX genes are different, implying that the functions of WOX genes in annual plants and woody plants might be different [8]. In A. thaliana, only WOX4 is involved in the differentiation of vascular meristem; however, poplars also need the WUS gene, as microtubule tissues are differentiated from the cambium every year in poplar $[16,61]$. The AtWOX5 gene is specifically expressed in QC cells, while the PtoWOX5 gene also can be found in the other tissues [44]. In this study, the expression patterns of WOX genes in walnut were more similar to those in poplar. The difference was that the PtoWOX3 gene was not identified in poplar, while $J r W O X 3 a$ and JrWOX3b were highly expressed in the leaves of walnut. PtoWOX11/12s and PtoWOX5s are uniquely expressed in roots; however, there also existed weak expression of JrWOX11 gene in other tissues besides the high expression in roots (Figure 4). It can be speculated that the functions of 
WOX genes in walnut might be more similar to poplar than annual plants. The differences between walnut and poplars might provide evidences that the rooting mechanisms of easy-to-root trees and difficult-to-root trees are different.

\subsection{Expression Profiling Revealed JrWOX Genes' Responses to Rejuvenation and Involvement in ARF}

Stem cuttings are the most extensively used method in woody plants. However, their capacity for forming adventitious roots becomes weaker as trees age. Some studies have shown that rejuvenation of mature trees or the induction of plants to regress from maturity to the juvenile state may significantly improve ARF. It might be owing to the changes of distribution of endogenous plant hormones after rejuvenation [5]. In this study, we further revealed the histological differences between rejuvenated and mature cuttings in walnut (Figure 5). Moreover, almost all the JrWOX genes were induced by being rejuvenated. This might be helpful to better understand the regulation mechanism of rejuvenation at the level of molecular.

The roles of WOX proteins in root formation and development have been well-documented in Arabidopsis, rice, tobacco, and poplar; for example, the leaf of Arabidopsis is used to study de novo root organogenesis, and the results show that AtWOX5 regulate stem cells in root apical meristem (RAM) and AtWOX11 mediate root primordium formation. In rice, OsWOX11 is required to activate lateral root initiation, root hair formation, and responses to abiotic stresses [24]. In poplar, over-expression of PtoWOX4, PtoWOX5a, PtoWOX11, and PtoWOX13 led to an increased AR number, decreased AR length, or increased AR roughness [21]. In tobacco, plant hormones, their intricate signaling networks and WOX play a crucial function in the process of ARF [62]. In this study, JrWOX5, JrWOX9, and JrWOX11 were strongly induced in adventitious root regeneration of rejuvenation cuttings, and JrWOX11 was also highly promoted in mature cuttings, implying JrWOX5, JrWOX9, and JrWOX11 play a pivotal part in ARF. JrWOX4 and JrWOX13 may play an important role in later root development, although the relative expression of them showed significant changes during ARF. It is unclear if the other WOX genes are involved in AFR, in view of the fact that there was no significant change in their expression level.

\section{Conclusions}

J. regia contains 12 WOX proteins and can be divided into three clades: the ancient, intermediate, and modern clades. The sequences of domains are relatively conservative, and the gene structure and motifs contribute to phylogenetic analysis of WOX genes. WOX genes may be recruited in root evolution. JrWOX4a/b, JrWOX11, and JrWOX13 were highly expressed in root, and JrWOX5 and JrWOX11 were strongly induced during adventitious root regeneration. Rejuvenation treatment reduced thickness and density of sclerenchyma between the cortex and phloem, and almost all JrWOX genes were expressed. This study also provides an approach for identifying and characterizing WOX genes in other species and reveals the possible functions of WOX genes in a difficult-to-root tree ARF. In future research, the rejuvenation function in ARF should be verified in more-difficult rooting species. The question of whether the hormone treatment for root induction is a standard treatment, and whether hormone treatment triggers the expression of JrWOX genes, should be focused on. Moreover, the genetic engineering means could be used to analyze the above genes' functions.

Author Contributions: Data curation, X.S.; formal analysis, Y.C.; funding acquisition, X.S., Q.Z., and D.P.; investigation, Y.B. and X.L.; resources, H.L.; supervision, D.P.; writing—original draft, Y.C.; writing一review and editing, X.S. All authors have read and agreed to the published version of the manuscript.

Funding: This research was funded by the National Key Research and Development Program of China (Grant: 2018YFD1000604), the National Natural Science Foundation of China (No. 31670682), and the National Natural Science Foundation of China (No. 31672126).

Conflicts of Interest: The authors declare no conflicts of interest. The funders had no role in the design of the study; in the collection, analyses, or interpretation of data; in the writing of the manuscript; or in the decision to publish. 


\section{Appendix A}

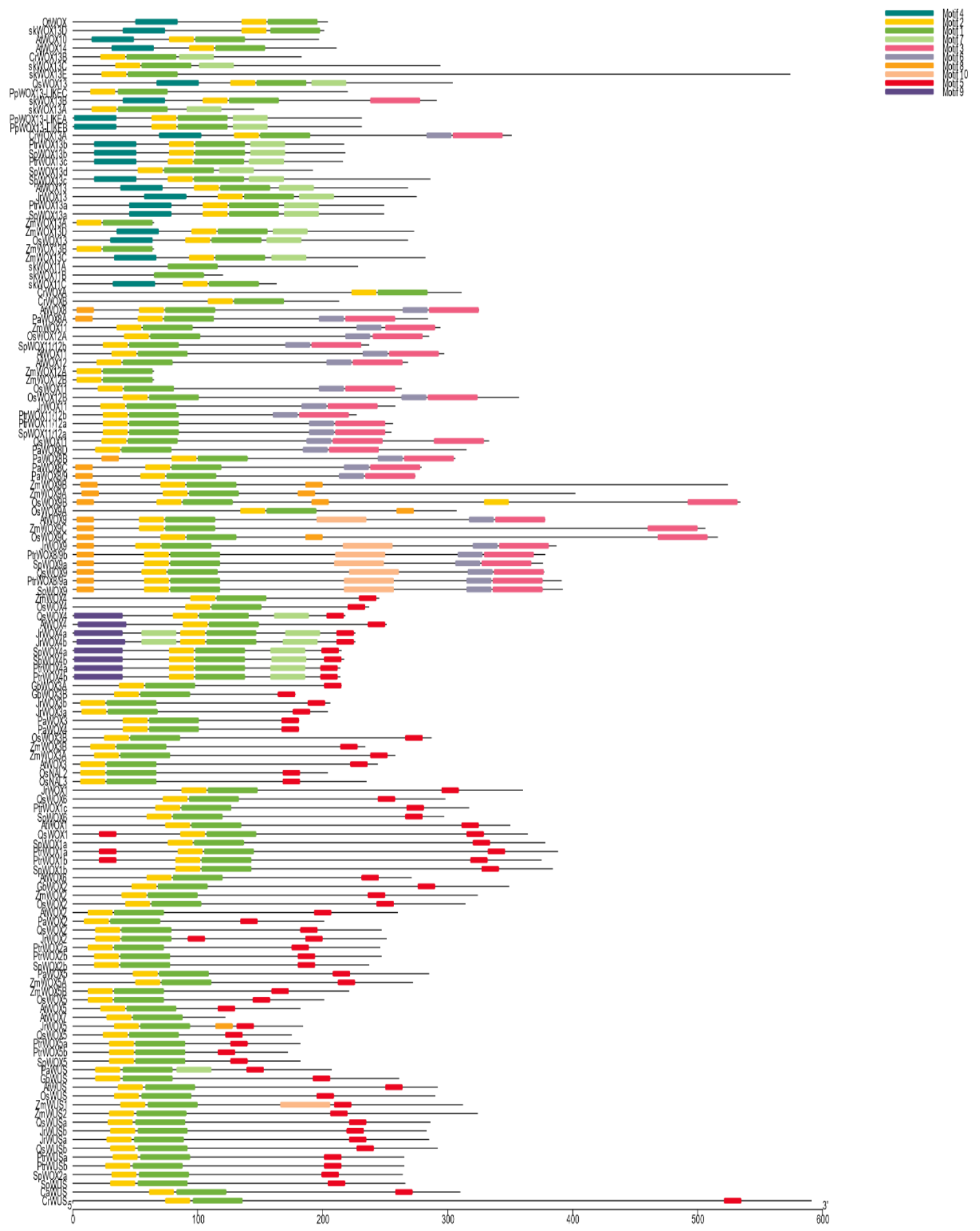

Figure A1. Motifs distribution of the whole protein sequences of OtWOXs, PpWOXs, CrWOXs, CaWOXs, GbWOXs, PaWOXs, AtWOXs, PtWOXs, ZmWOXs, OsWOXs, and SpWUS. QsWOXs and JrWOXs. 


\section{Appendix B}

Table A1. Accession numbers and genome loci of the WOX sequences used for phylogenetic analysis. Ot: Ostreococcus tauri, Pp: Physcomitrella patens, Sk: Selaginella kraussiana, Cr: Ceratopteris richardii, Ca: Cyathea australis, Gb: Ginkgo biloba, Pa: Picea abies, Os: Oryza sativa, Zm: Zea mays, At: Arabidopsis thaliana, Ptr: Populus trichocarpa, Sp: Spirodela polyrhiza, Qs: Quercus suber and Jr: Juglans regia [63-66].

\begin{tabular}{|c|c|c|c|}
\hline Gene Name & Accession No./Locus & Gene Name & Accession No. \\
\hline Pa_WUS & JX512364 & Zm_WUS1 & AM234744 \\
\hline Pa_WOX2 & AM286747 & Zm_WUS2 & AM234745 \\
\hline Pa_WOX3 & JX411947 & Zm_WOX2 & AM490235 \\
\hline Pa_WOX4 & JX411948 & Zm_WOX3B & AM491777 \\
\hline Pa_WOX5 & JX411949 & Zm_WOX3A & AM490236 \\
\hline $\mathrm{Pa} \_\mathrm{WOX} 8 / 9$ & GU944670 & Zm_WOX4 & AM490237 \\
\hline Pa_WOX8A & JX411950 & Zm_WOX5A & AM490238 \\
\hline Pa_WOX8B & JX411951 & Zm_WOX5B & AM490239 \\
\hline Pa_WOX8C & JX411952 & Zm_WOX9A & AM490240 \\
\hline Pa_WOX8D & JX411953 & Zm_WOX9B & AM490241 \\
\hline At_WOX1 & AT3G18010 & Zm_WOX9C & AM490242 \\
\hline At_WOX2 & AT5G59340 & Zm_WOX11 & EU954172 \\
\hline At_WOX3 & AT2G28610 & Zm_WOX12A & AM234774 \\
\hline At_WOX4 & AT1G46480 & Zm_WOX12B & AM234775 \\
\hline At_WOX7 & AT5G05770 & Zm_WOX13A & AM234776 \\
\hline At_WOX5 & AT3G11260 & Zm_WOX13B & AM234777 \\
\hline At_WOX6 & AT2G01500 & Zm_WOX13C (ZmWOX14B) & EU952747 \\
\hline At_WUS & AT2G17950 & Zm_WOX13D (ZmWOX14A) & EU961475 \\
\hline At_WOX9 & AT2G33880 & Vv_WUS & AM447418 \\
\hline At_WOX8 & AT5G45980 & Vv_WOX1 & AM439847 \\
\hline At_WOX11 & AT3G03660 & Vv_WOX2 & AM488389 \\
\hline At_WOX12 & AT5G17810 & Vv_WOX3 & AM429035 \\
\hline At_WOX13 & AT4G35550 & Vv_WOX4 & AM447494 \\
\hline At_WOX10 & AT1G20710 & Vv_WOX5 & AM454567 \\
\hline At_WOX14 & AT1G20700 & Vv_WOX6 (VvWOX1B) & AM463144 \\
\hline Gb_WUS & FM882128 & Vv_WOX9 & AM488026 \\
\hline Gb_WOX2 & FM882124 & Vv_WOX11 & AM435207 \\
\hline Gb_WOX3A & FM882125 & Vv_WOX13A & AM486367 \\
\hline Gb_WOX3B & FM882126 & Vv_WOX13B & AM473516 \\
\hline Pp_WOX13-LIKE A & AB699867 & Vv_WOX13C & AM463736 \\
\hline Pp_WOX13-LIKE B & AB699868 & Sp_WOX4A & KF982703 \\
\hline Pp_WOX13-LIKE C & XM_024511279 & Sp_WOX4B & KC691250 \\
\hline Sm_WOX13 & XM_002962367 & Sp_WOX1B & KF982700 \\
\hline Sm_WOX8 & XM_024668721 & Sp_WOX1A & KF982699 \\
\hline Sm_WOX10 & XM_002981839 & Sp_WOX9A & KF982708 \\
\hline Sm_WOX11 & XM_024684472 & Sp_WOX13B & KF982713 \\
\hline Sm_WOX6 & XM_024673314 & Sp_WOX13C & AJ010810 \\
\hline Sm_WOX5 & EFJ20992 & Sp_WOX13D(8) & AY479970 \\
\hline Sm_WOX9 & EFJ33362 & Sp_WOX13A & KF982712 \\
\hline Sm_WOX1 & EFJ33357 & Sp_WOX6 & KF982707 \\
\hline Sm_WOX3 & EFJ33027 & Sp_WOX11/12B & KF982709 \\
\hline Sm_WOX4 & EFJ21095 & Sp_WOX11/12A & KF982710 \\
\hline Sm_WOX2 & EFJ35854 & Sp_WUSA & KF982698 \\
\hline Ca_WUS & FR716459 & Sp_WOX2B & KF982702 \\
\hline Ca_WOXA & FR716461 & Sp_WOX2A & FJ232064 \\
\hline Ca_WOX13 & FR716460 & Sp_WOX5B & KF982706 \\
\hline Cr_WUS & FR716458 & Sp_WOX9B & KF982709 \\
\hline Cr_WOXA & FR716456 & PtrWUSa & Potri.005G114700 \\
\hline
\end{tabular}


Table A1. Cont.

\begin{tabular}{cccc}
\hline Gene Name & Accession No./Locus & Gene Name & Accession No. \\
\hline Cr_WOXB & FR716457 & PtrWUSb & Potri.007G012100 \\
Cr_WOX13A & FR716454 & PtrWOX1a & Potri.012G047700 \\
Cr_WOX13B & FR716455 & PtrWOX1b & Potri.015G039100 \\
Os_WOX3a & LOC_Os11g01130.1 & PtrWOX1c & Potri.010G111400 \\
Os_WOX3b & LOC_Os12g01120.1 & PtrWOX2a & Potri.001G237900 \\
Os_WOX3c & LOC_Os11g01130.2 & PtrWOX2b & Potri.009G029200 \\
Os_WOX2 & LOC_Os05g02730.1 & PtrWOX4a & Potri.002G124100 \\
Os_WOX4 & LOC_Os04g55590.1 & PtrWOX4b & Potri.014G025300 \\
Os_WOX5 & LOC_Os01g62310.1 & PtrWOX5a & Potri.008G065400 \\
Os_WOX1 & LOC_Os04g56780.1 & PtrWOX5b & Potri.010G192100 \\
OsWOX9 & LOC_Os01g63510.1 & PtrWOX8/9a & Potri.004G051600 \\
OsWOX7 & LOC_Os01g47710.1 & PtrWOX8/9b & Potri.011G061400 \\
OsWOX12 & LOC_Os05g48990.1 & PtrWOX11/12a & Potri.013G066900 \\
OsWOX11 & LOC_Os07g48560.1 & PtrWOX11/12b & Potri.019G040800 \\
OsWOX6 & LOC_Os03g20910.1 & PtrWOX13a & Potri.005G101800. \\
OsWOX13 & LOC_Os07g34880.1 & PtrWOX13b & Potri.005G252800 \\
OsWOX10 & LOC_Os08g14400.1 & PtrWOX13c & Potri.002G008800 \\
OsWOX8 & LOC_Os01g60270.1 & & \\
\hline
\end{tabular}

Table A2. The primer sequences of qRT-PCR.

\begin{tabular}{cccc}
\hline Genes & Primer Sequence $\left(\mathbf{F}: \mathbf{5}^{\prime} \rightarrow \mathbf{3}^{\prime}\right)$ & Primer Sequence $\left(\mathbf{F}: \mathbf{5}^{\prime} \rightarrow \mathbf{3}^{\prime}\right)$ & Production/bp \\
\hline GAPDH & ATGATGTCAAGGAAGGACTC & CACAATGATCTCAGCTCCG & 102 \\
$J r W U S a$ & ACTCCTTCATCCAACGGCTT & TGAGCTCCAGGGAAGTACGA & 88 \\
$J r W U S b$ & TCAATGGCTTCTGCAATGTTAG & AGAACCGCTCTTGCCACTGT & 104 \\
$J r W O X 1$ & ACGAACACCAAAGTGCCCAT & ATCGGCTGGCTGTTTCCT & 105 \\
$J r W O X 2$ & CTCACGCTGGAATCCGACA & CAATGTGACCGTAAGCCCG & 128 \\
$J r W O X 3 a$ & ACGGCTATGATGGGAATGCTA & AACAGGGAAGAGTTCAAGGGTC & 92 \\
$J r W O X 3 b$ & CTGCTCTTCAACAACTTTCCCA & TTTGAGTTGTCTATCTCGCCCA & 157 \\
$J r W O X 4 a$ & CAAGCAGGCTAACTCCGACA & GGGGAATCTCGTTTGCGTC & 126 \\
$J r W O X 4 b$ & AGCACGAACCCTCCCTCAC & AAAAGCCGCAACACCAGC & 106 \\
$J r W O X 5$ & GACCCTCCAACTTTCCCATT & AGTGCTCCATTTCTGTCCCAA & 122 \\
$J r W O X 9$ & ACACAACTGCGGTCTCTCCCT & ACCCGATTCCCCAACACCT & 95 \\
$J r W O X 11$ & GACGTAGTGTTGGTCCATTGCT & GCCCTCTTTCTTCCCCTTTAA & 135 \\
$J r W O X 13$ & CAGGGCTGAAGATTTGTGCT & TACTGCCATCTGACGGGAAC & 107 \\
\hline
\end{tabular}

\section{References}

1. Bonga, J.M. Vegetative Propagation in Relation to Juvenility, Maturity, and Rejuvenation. In Tissue Culture in Forestry; Springer: Berlin/Heidelberg, Germany, 1982; pp. 387-412.

2. Hackett, W.P.; Murray, J.R. Maturation and rejuvenation in woody species. In Micropropagation of Woody Plants; Springer: Berlin/Heidelberg, Germany, 1993; pp. 93-105.

3. Wendling, I.; Trueman, S.J.; Xavier, A. Maturation and related aspects in clonal forestry-Part II: Reinvigoration, rejuvenation and juvenility maintenance. New For. 2014, 45, 473-486. [CrossRef]

4. Von Aderkas, P.; Bonga, J.M. Influencing micropropagation and somatic embryogenesis in mature trees by manipulation of phase change, stress and culture environment. Tree Physiol. 2000, 20, 921-928. [CrossRef] [PubMed]

5. Liu, H.; Gao, Y.; Song, X.B.; Ma, Q.G.; Zhang, J.P.; Pei, D. A novel rejuvenation approach to induce endohormones and improve rhizogenesis in mature Juglans tree. Plant Methods 2018, 14, 13. [CrossRef] [PubMed]

6. Suklabaidya, A.; Mehta, K. Rejuvenation of Senile Horticultural Plantations for Improved Productivity and Quality. Int. J. Hortic. Agric. Food Sci. 2019, 3, 4. [CrossRef]

7. Chang, F.; Chen, P.-J.; Shen, C.-H.; Hsieh, T.-J.; Hsu, Y.-W.; Huang, B.-L.; Kuo, C.-I.; Chen, Y.-T.; Chu, H.-A.; Yeh, K.-W.; et al. Proteomic profiling of proteins associated with the rejuvenation of Sequoia sempervirens (D. Don) Endl. Proteome Sci. 2010, 8, 64. [CrossRef] 
8. Van Der Graaff, E.; Laux, T.; Rensing, S.A. The WUS homeobox-containing (WOX) protein family. Genome Biol. 2009, 10, 248. [CrossRef]

9. Liu, B.; Wang, L.; Zhang, J.; Li, J.B.; Zheng, H.Q.; Chen, J.; Lu, M.Z. WUSCHEL-related Homeobox genes in Populus tomentosa: Diversified expression patterns and a functional similarity in adventitious root formation. BMC Genom. 2014, 15, 296. [CrossRef]

10. Lian, G.; Ding, Z.W.; Wang, Q.; Zhang, D.B.; Xu, J. Origins and evolution of WUSCHEL-related homeobox protein family in plant kingdom. Sci. World J. 2014, 2014, 1-13. [CrossRef]

11. Yves, D.; Claire, T.N.; Gaelle, C.; Vincent, T.; Halima, M.; Patrick, L.; Hervé, M.; Martin, K.; Alain, L. Genes of the most conserved WOX clade in plants affect root and flower development in Arabidopsis. BMC Evol. Biol. 2008, 8, 291.

12. Zhang, X.; Zong, J.; Liu, J.H.; Yin, J.Y.; Zhang, D.B. Genome-Wide Analysis of WOX Gene Family in Rice, Sorghum, Maize, Arabidopsis and Poplar. J. Integr. Plant Biol. 2010, 52, 1016-1026. [CrossRef]

13. Haecker, A.; Groß-hardt, R.; Geiges, B.; Sarkar, A.; Breuninger, H.; Herrmann, M.; Laux, T. Expression dynamics of WOX genes mark cell fate decisions during early embryonic patterning in Arabidopsis thaliana. Development 2004, 131, 657-668. [CrossRef] [PubMed]

14. Schoof, H.; Lenhard, M.; Haecker, A.; Mayer, K.F.X.; Laux, T. The Stem Cell Population of Arabidopsis Shoot Meristems is maintained by a regulatory loop between the CLAVATA and WUSCHEL genes. Cell 2000, 100, 635-644. [CrossRef]

15. Luijten, M.; Miyashima, S.; Lenhard, M.; Hashimoto, T.; Nakajima, K.; Scheres, B.; Heidstra, R.; Laux, T.; Sarkar, A.K. Conserved factors regulate signalling in Arabidopsis thaliana shoot and root stem cell organizers. Nature 2007, 446, 811-814.

16. Stahl, Y.; Wink, R.H.; Ingram, G.C.; Simon, R. A signaling module controlling the stem cell niche in Arabidopsis root meristems. Curr. Biol. 2009, 19, 909-914. [CrossRef] [PubMed]

17. Hirakawa, Y.; Kondo, Y.; Fukuda, H. TDIF Peptide Signaling Regulates Vascular Stem Cell Proliferation via the WOX4 Homeobox Gene in Arabidopsis. Plant Cell 2010, 22, 2618-2629. [CrossRef] [PubMed]

18. Shimizu, R.; Ji, J.; Kelsey, E.; Ohtsu, K.; Schnable, P.S.; Scanlon, M.J. Tissue Specificity and Evolution of Meristematic WOX3 Function. Plant Physiol. 2009, 149, 841-850. [CrossRef] [PubMed]

19. Breuninger, H.; Rikirsch, E.; Hermann, M.; Ueda, M.; Laux, T. Differential expression of WOX genes mediates apical-basal axis formation in the Arabidopsis embryo. Dev. Cell 2008, 14, 867-876. [CrossRef] [PubMed]

20. Park, S.O.; Zheng, Z.; Oppenheimer, D.G.; Hauser, B.A. The PRETTY FEW SEEDS2 gene encodes an Arabidopsis homeodomain protein that regulates ovule development. Development 2005, 132, 841-849. [CrossRef]

21. Wu, X.; Chory, J.; Weigel, D. Combinations of WOX activities regulate tissue proliferation during Arabidopsis embryonic development. Dev. Biol. 2007, 309, 306-316. [CrossRef]

22. Liu, J.; Sheng, L.; Xu, Y.; Li, J.; Yang, Z.; Huang, H.; Xu, L. WOX11 and 12 Are Involved in the First-Step Cell Fate Transition during de Novo Root Organogenesis in Arabidopsis. Plant Cell 2014, 26, 1081-1093. [CrossRef]

23. Liu, J.; Hu, X.; Qin, P.; Prasad, K.; Hu, Y.; Xu, L. The WOX11—LBD16 Pathway Promotes Pluripotency Acquisition in Callus Cells during de Novo Shoot Regeneration in Tissue Culture. Plant Cell Physiol. 2018, 59, 734-743. [CrossRef] [PubMed]

24. Tian, H.; Jia, Y.; Niu, T.; Yu, Q.; Ding, Z. The key players of the primary root growth and development also function in lateral roots inArabidopsis. Plant Cell Rep. 2014, 33, 745-753. [CrossRef] [PubMed]

25. Zhao, Y.; Hu, Y.; Dai, M.; Huang, L.; Zhou, D.-X. The WUSCHEL-Related Homeobox Gene WOX11 Is Required to Activate Shoot-Borne Crown Root Development in Rice. Plant Cell Online 2009, 21, 736-748. [CrossRef] [PubMed]

26. Zhao, Y.; Cheng, S.; Song, Y.; Huang, Y.; Zhou, S.; Liu, X.; Zhou, D.-X. The Interaction between Rice ERF3 and WOX11 Promotes Crown Root Development by Regulating Gene Expression Involved in Cytokinin Signaling. Plant Cell 2015, 27, 2469-2483. [CrossRef]

27. Jiang, W.; Zhou, S.; Zhang, Q.; Song, H.; Zhou, D.-X.; Zhao, Y. Transcriptional regulatory network of WOX11 is involved in the control of crown root development, cytokinin signals, and redox in rice. J. Exp. Bot. 2017, 68, 2787-2798. [CrossRef]

28. Xu, M.; Xie, W.F.; Huang, M.R. Two WUSCHEL-related HOMEOBOX genes, PeWOX11a and PeWOX11b, are involved in adventitious root formation of poplar. Physiol. Plant. 2015, 155, 446-456. [CrossRef] 
29. Stevens, M.E.; Woeste, K.E.; Pijut, P.M. Localized gene expression changes during adventitious root formation in black walnut (Juglans nigra L.). Tree Physiol. 2018, 38, 877-894. [CrossRef]

30. Song, X.B.; Xi, S.K.; Zhang, J.P.; Ma, Q.G.; Zhou, Y.; Xu, H.; Zhang, J.W.; Pei, D. 'ZhongNingsheng': A New Distant Hybrid Cultivar of Walnut. Hortscience 2019, 54, 2257-2259. [CrossRef]

31. Pei, D.; Gu, R.S. Shoot Rooting in vitro for Walnut Cultivars. Sci. Silvae Sin. 2002, 38, 32-37.

32. Pei, D.; Zhang, J.P.; Xu, H.Z. Cutting Propagation Method for Juglans: China. CN 2004100092012005 , 11 June 2004.

33. Ma, Q.; Zhang, J.; Pei, D. Genetic Analysis of Walnut Cultivars in China Using Fluorescent Amplified Fragment Length Polymorphism. J. Am. Soc. Hortic. Sci. 2019, 136, 422-428. [CrossRef]

34. Song, X.B.; Sun, L.; Luo, H.T.; Ma, Q.G.; Zhao, Y.; Pei, D. Genome-wide identification and characterization of long non-coding RNAs from mulberry (Morus notabilis) RNA-Seq data. Genes 2016, 7, 11. [CrossRef] [PubMed]

35. Song, X.B.; Chang, Y.Y.; Liu, H.; Xu, H.M.; Pei, D. Reference Gene Selection and Genes Expression Analysis During Adventitious Root Formation in Walnut. Acta Hortic. Sin. 2019, 46, 1907-1918. [CrossRef]

36. Martínezgarcía, P.J.; Crepeau, M.W.; Puiu, D.; Gonzalezibeas, D.; Whalen, J.; Stevens, K.A.; Paul, R.; Butterfield, T.S.; Britton, M.T.; Reagan, R.L. The walnut (Juglans regia) genome sequence reveals diversity in genes coding for the biosynthesis of non-structural polyphenols. Plant J. Cell Mol. Biol. 2016, 87, 507-532. [CrossRef] [PubMed]

37. Mark, J.; Irena, Z.; Yan, R.; Yuri, M.; Scott, M.; Madden, T.L. NCBI BLAST: A better web interface. Nucleic Acids Res. 2008, 36, W5-W9.

38. Larkin, M.A.; Blackshields, G.; Brown, N.P.; Chenna, R.; Mcgettigan, P.A.; Mcwilliam, H.; Valentin, F.; Wallace, I.M.; Wilm, A.; Lopez, R. Clustal W and Clustal X version 2.0. Bioinformatics 2007, 23, 2947-2948. [CrossRef]

39. Kumar, S.; Stecher, G.; Tamura, K. MEGA7: Molecular Evolutionary Genetics Analysis Version 7.0 for Bigger Datasets. Mol. Biol. Evol. 2016, 33, 1870. [CrossRef]

40. Pfaffl, M.W.; Hageleit, M. Validities of mRNA quantification using recombinant RNA and recombinant DNA external calibration curves in real-time RT-PCR. Biotechnol. Lett. 2001, 23, 275-282. [CrossRef]

41. Mukherjee, K.; Brocchieri, L.; Burglin, T.R. A Comprehensive Classification and Evolutionary Analysis of Plant Homeobox Genes. Mol. Biol. Evol. 2009, 26, 2775-2794. [CrossRef]

42. Liu, W.; Xu, L. Recruitment of IC-WOX Genes in Root Evolution. Trends Plant Sci. 2018, 23, $490-496$. [CrossRef]

43. Zeng, M.; Hu, B.; Li, J.; Zhang, G.; Xu, L. Stem cell lineage in body layer specialization and vascular patterning of rice root and leaf. Sci. Bull. 2016, 61, 847-858. [CrossRef]

44. Li, J.; Zhang, J.; Jia, H.; Liu, B.; Sun, P.; Hu, J.; Wang, L.; Lu, M. The WUSCHEL-related homeobox 5a (PtoWOX5a) is involved in adventitious root development in poplar. Tree Physiol. 2017, 38, 1-15. [CrossRef] [PubMed]

45. Bi, C.; Xu, Y.; Ye, Q.; Yin, T.; Ye, N. Genome-wide identification and characterization of WRKY gene family in Salix suchowensis. PeerJ 2016, 4, e2437. [CrossRef] [PubMed]

46. Lin, H.; Niu, L.; McHale, N.A.; Ohme-Takagi, M.; Mysore, K.S.; Tadege, M. Evolutionarily conserved repressive activity of WOX proteins mediates leaf blade outgrowth and floral organ development in plants. Proc. Natl. Acad. Sci. USA 2013, 110, 366-371. [CrossRef] [PubMed]

47. Romerabranchat, M.; Ripoll, J.J.; Yanofsky, M.F.; Pelaz, S. The WOX13 homeobox gene promotes replum formation in the Arabidopsis thaliana fruit. Plant J. 2013, 73, 37-49. [CrossRef] [PubMed]

48. Chen, X.; Qu, Y.; Sheng, L.; Liu, J.; Huang, H.; Xu, L. A simple method suitable to study de novo root organogenesis. Front. Plant Sci. 2014, 5, 1-6. [CrossRef] [PubMed]

49. Kong, D.; Hao, Y.; Cui, H. The WUSCHEL Related Homeobox Protein WOX7 Regulates the Sugar Response of Lateral Root Development in Arabidopsis thaliana. Mol. Plant. 2016, 9, 261-270. [CrossRef]

50. Nardmann, J.; Werr, W. The invention of WUS-like stem cell-promoting functions in plants predates leptosporangiate ferns. Plant Mol. Biol. 2012, 78, 123-134. [CrossRef]

51. Rodriguez, K.; Perales, M.; Snipes, S.; Yadav, R.K.; Diaz-Mendoza, M.; Reddy, G.V. DNA-dependent homodimerization, sub-cellular partitioning, and protein destabilization control WUSCHEL levels and spatial patterning. Proc. Natl. Acad. Sci. USA 2016, 113, E6307-E6315. [CrossRef]

52. Lu, G.; Bruce, W.B. A novel cis-acting element conferring root-preferred gene expression in maize. J. Plant Physiol. 2000, 156, 277-283. [CrossRef] 
53. Li, Z. Functional Charaterization of a PtoWOX11/12a gene in Stress Resistance of Populus tomentosa. Ph.D. Thesis, Chinese Academy of Forestry, Beijing, China, 2017.

54. Caboni, E.; Tonelli, M.G.; Lauri, P.; Iacovacci, P.; Kevers, C.; Damiano, C.; Gaspar, T. Biochemical aspects of almond microcuttings related to in vitro rooting ability. Biol. Plant. 1997, 39, 91-97. [CrossRef]

55. Berkefeld, H. Pyk10, a seedling and root specific gene and promoter from Arabidopsis thaliana. Plant Sci. 2001, 161, 337-346.

56. He, P.; Zhang, Y.; Liu, H.; Yuan, Y.; Wang, C.; Yu, J.; Xiao, G. Comprehensive analysis of WOX genes uncovers that WOX13 is involved in phytohormone-mediated fiber development in cotton. BMC Plant Biol. 2019, 19, 312. [CrossRef] [PubMed]

57. Amissah, J.N.; Paolillo, D.J.; Bassuk, N. Adventitious root formation in stem cuttings of Quercus bicolor and Quercus macrocarpa and its relationship to stem anatomy. J. Am. Soc. Hortic. Sci. 2008, 133, 479-486. [CrossRef]

58. Liu, B.; Zhang, J.; Yang, Z.; Matsui, A.; Seki, M.; Li, S.; Yan, X.; Kohnen, M.V.; Gu, L.; Prasad, K.; et al. PtWOX11 acts as master regulator conducting the expression of key transcription factors to induce de novo shoot organogenesis in poplar. Plant Mol. Biol. 2018, 98, 389-406. [CrossRef]

59. Edwards, R.A.; Thomas, M.B. Observations on physical barriers to root formation in cuttings. Plant Propagator 1980, 26, 6-8.

60. Amissah, J.N.; Bassuk, N. Cutting back stock plants promotes adventitious rooting of stems of Quercus bicolor and Quercus macrocarpa. J. Environ. Hortic. 2009, 73, 159-165.

61. Kucukoglu, M.; Nilsson, J.; Zheng, B.; Chaabouni, S.; Nilsson, O. WUSCHEL-RELATED HOMEOBOX4 (WOX4)-like genes regulate cambial cell division activity and secondary growth in Populus trees. New Phytol. 2017, 215, 642-657. [CrossRef]

62. Druege, U.; Franken, P.; Hajirezaei, M.R. Plant Hormone Homeostasis, Signaling, and Function during Adventitious Root Formation in Cuttings. Front. Plant Sci. 2016, 7, 381. [CrossRef]

63. Cho, S.H.; Paek, N.C. Regulatory role of the OsWOX3A transcription factor in rice root development. Plant Signal. Behav. 2016, 11, e1184807. [CrossRef]

64. Pi, L.; Aichinger, E.; van der Graaff, E.; Llavata-Peris, C.I.; Weijers, D.; Hennig, L.; Groot, E.; Laux, T. Organizer-Derived WOX5 Signal Maintains Root Columella Stem Cells through Chromatin-Mediated Repression of CDF4 Expression. Dev. Cell 2015, 33, 576-588. [CrossRef]

65. Chen, G.; Feng, H.; Hu, Q.; Qu, H.; Chen, A.; Yu, L.; Xu, G. Improving rice tolerance to potassium deficiency by enhancing OsHAK16p: WOX11-controlled root development. Plant Biotechnol. J. 2015, 13, 833-848. [CrossRef] [PubMed]

66. Tang, F.; Chen, N.; Zhao, M.; Wang, Y.; He, R.; Peng, X.; Shen, S. Identification and functional divergence analysis of WOX gene family in paper mulberry. Int. J. Mol. Sci. 2017, 18, 1782. [CrossRef] [PubMed] 